\title{
MISSION-ORIENTED SENSOR ARRAYS AND UAVs - A CASE STUDY ON ENVIRONMENTAL MONITORING
}

\author{
N. M. Figueira ${ }^{\text {a,c }}$, I. L. Freire ${ }^{\text {b }}$, O. Trindade ${ }^{c}$, E. Simões ${ }^{c}$ \\ a Brazilian Army, Dept. of Science and Technology,Quartel-General do Exército - Bloco G - $3^{\circ}$ Andar,Brasília, Brasil - \\ nina.figueira@dsg.eb.mil.br \\ b Dept. of Computer Science, Minas Gerais Federal University, Av. Antônio Carlos, 6627, Belo Horizonte, Brazil - \\ izabela@dcc.ufmg.br \\ c São Paulo University, São Paulo, Av. Trabalhador São Carlense, São Carlos, 400, Brazil - otj@icmc.usp.br; edvsimoes@gmail.com
}

\section{Commission VI, WG VI/4}

KEY WORDS: MOSA, Sound Source Localization, Thematic Maps, Multi-Sensor Data Fusion, Model Driven Development, Environmental Monitoring.

\begin{abstract}
:
This paper presents a new concept of UAV mission design in geomatics, applied to the generation of thematic maps for a multitude of civilian and military applications. We discuss the architecture of Mission-Oriented Sensors Arrays (MOSA), proposed in Figueira et Al. (2013), aimed at splitting and decoupling the mission-oriented part of the system (non safety-critical hardware and software) from the aircraft control systems (safety-critical). As a case study, we present an environmental monitoring application for the automatic generation of thematic maps to track gunshot activity in conservation areas. The MOSA modeled for this application integrates information from a thermal camera and an on-the-ground microphone array. The use of microphone arrays technology is of particular interest in this paper. These arrays allow estimation of the direction-of-arrival (DOA) of the incoming sound waves. Information about events of interest is obtained by the fusion of the data provided by the microphone array, captured by the UAV, fused with information from the termal image processing. Preliminary results show the feasibility of the on-the-ground sound processing array and the simulation of the main processing module, to be embedded into an UAV in a future work. The main contributions of this paper are the proposed MOSA system, including concepts, models and architecture.
\end{abstract}

\section{INTRODUCTION}

Unmanned Aerial Vehicles (UAV) have been increasingly used in applications such as agriculture management, wildlife research, environmental monitoring and natural or manmade disaster supervising. In the military scenario, the use of UAVs has focused traditionally on the accomplishment of specific tasks in two broad categories: remote sensing and transport of military material.

The Mission-Oriented Sensor Array (MOSA) architecture is discussed in this paper. In this architecture, the UAV and its control system are considered as a transportation platform, able to carry the MOSA payload to specific locations in the area of interest. The MOSA payload communicate with the control system of the aircraft through the Smart Sensor Protocol (SSP) (Pires, 2014), specifically developed for this task. In this sense, different missions can be performed by the same UAV exchanging the MOSA payload. Moreover, the MOSA concept reduces or eliminates the requirements on high bandwidth communication channels to ground facilities normally used to carry real time data such as high resolution images.

Different processing tasks and different sensors can be integrated into a MOSA payload allowing for the best arrangement for each usage scenario. Among them can be mentioned: thermal cameras, microphones, RGB cameras, LIDAR (Light Detection And Ranging), SAR (Synthetic Aperture Radar), high precision Global Positioning System (GPS) receivers and Inertial Measurement Units (IMU). These sensors allow direct georeferencing and the production of ready to use thematic maps. Furthermore, MOSA systems can be adaptive in real time, dealing with mission changes that may be due to bad weather conditions or moving subjects. It is also possible to request reconfiguration of the sensor arrays to fit certain mission characteristics.

This work is related to the "Ranger Drone Project" (Hemav Academics, 2014). In that project, the MOSA architecture was applied to an environmental preservation project in the Kruger National Park in South Africa. That is referred to as the Ranger's MOSA (R_MOSA) and integrates audio and video information about poaching and wildlife trafficking to support ranger's activity in wildlife preservation. The MOSA scanning process is completely separated from the aircraft flight control system. Its objective is to detect any possible threat and send a warning along with photographies of the area, so the ranger can decide whether it is a real threat or just a false alarm.

The structure of the remaining text is: Section 2 presents the MOSA system architecture; Section 3 introduces audio signal processing; Section 4 describes the proposed case study; Section 5 presents some preliminary results; Section 6 addresses discussions and future works and Section 7 presents the conclusions.

\section{MOSA SYSTEM ARCHTECTURE}

\subsection{Unmanned Aerial Systems}

In 2009 the US Department of Defense - DOD, followed by the Federal Aviation Administration - FAA and the European Aviation Safety Agency- EASA, widened the UAV (Unmanned Aerial Vehicle) concept to the UAS concept (Unmanned Aircraft System). According to (Austin, 2010), UAVs have advantage over manned aircraft when applied to Dirty, Dull and Dangerous (DDD) missions.

According to Austin (2010), a UAS is constituted by: 


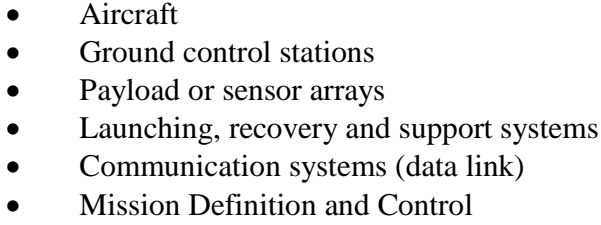

2.1.1 Aircraft: The Aircraft is the aerial vehicle together with its propulsion and fuel. There can be as many aircraft as it is necessary to keep continuous operation, what depends on flight autonomy and time (Austin, 2010). Most of the systems employ up to three identical aircraft. The aircraft can be classified according to its functional category, size, cargo capacity, range, autonomy, among others. According to (Longhitano, 2010) an UAS can be classified as: HALE (High altitude long endurance); MALE (Medium altitude long endurance); TUAV (Tactical); MUAV (Mini); MAV (Micro); NUAV (Nano).

It is estimated that the great majority of civil UAS will be constituted by small aircrafts ad payloads (Mini-UAS), with low cost, small weight and with maximum independency in relation to aerial infrastructure (such as airports).

2.1.2 Ground Control Stations: The ground control station or CGS is a ground-based computer system that monitors the mission, receiving data from the aircraft embedded sensors (such as fuel level, altitude, and airspeed). It should also be able to manage the communication system. The security of this data link is a fundamental factor to be considered. The GCS may vary from simple smartphones, tablets or notebooks to more complex systems constituted by computers, flight control stations and communication systems, among others.

Most of GCS perform the following functions:

- Aircraft monitoring and control (manual and autonomous);

- Payload monitoring and control: management of data obtained from the sensors or control of the embedded weapons.

According to (Trindade et al., 2010), usually trained personal using supervised and non-supervised processing algorithms are nowadays responsible for data processing on the ground in a GCS. In some cases, there is manual inspection of the data but this not always comply with the temporal resolution requirements of the application. A good example is data processing for agriculture management, where the detection of plagues and diseases must generate thematic maps within a specific time slot otherwise the crop can be compromised before the application of the appropriate countermeasures. Automatic data processing sounds promising in this scenario.

2.1.3 Communication systems: According to (Pastor et al., 2007), a communication system is a hardware and software architecture that allows the transmission of data and messages between aircraft and GCS.

The communication system allows integration with other systems, involving Command, Control, Communication, Computation, Intelligence, Information, Surveillance, and Recognition $\left(\mathrm{C}_{4} \mathrm{I}_{2} \mathrm{SR}\right)$. It consists of all necessary equipment to perform the flight control data link, to transmit payload data, and to coordinate aerial traffic.
2.1.4 Payloads: Payloads are the embedded operational devices dedicated to the mission (sensors, cameras, among others). According to Austin (2010), the kind and performance of the payload are defined according to the mission specific needs. These systems can be simple, such as a small nonstabilized video camera, or more sophisticated systems, such as gyro-stabilized cameras or even a high power radar.

2.1.5 Launching, recovery and support systems: These systems consist of the mechanisms to accommodate and transport the many parts of the UAS, its launching platform, the recovery equipment, and their maintenance tools.

2.1.6 Mission Definition and Control: Nowadays, mission definitions, aircraft control in case of an emergency and mission modification are performed manually by operational specialists that constitute the GCS team. According to (Pastor et al., 2007), mission definition is associated to the navigation control system. This system is composed of an array of sensors designed to collect aerodynamics and positional data, such as GPS, compass, accelerometer, pressure, and gyroscope, among others. These data is sent to the flight computer that controls the motor and servos, commanding the aircraft according to the flight plan.

\subsubsection{New UAS Architectures: New requirements for UAS} design involve:

- Separation of mission from control;

- Improvements on in-flight awareness to overcome the lack of an on-board human pilot;

- Improvements on flight safety to avoid air-to-air and air-toground accidents;

The development of the control systems of an unmanned aircraft must follow safety-critical methodologies and be certified under strict standards such as the DO-178C. On the other hand, the mission software can be mission-critical but must not interfere with the safety-critical nature of the entire system. Separating mission systems from control systems helps to achieve this goal. This is the main reason for MOSA. Besides that the MOSA concept makes easier to adapt the aircraft for different missions and the development of the non-safetycritical mission related systems.

An on-board human pilot has an important role in maintaining flight safety. For unmanned aircraft, this role is transferred for an on-the-ground operator that do not have the same consciousness. In this work, as it was already proposed in (Rodrigues et al., 2011) and (Mattei, 2013), this ability is called In-flight Awareness (IFA). A human pilot can notice strange smells or vibrations, hear non-habitual noises, evaluate cloud formations, as well as be aware of political borders and the characteristics of the terrain. All those knowledge can be utilized to avoid or mitigate dangerous situations and select the best emergency protocols to use.

When interconnected, the aircraft and MOSA communicate using a Smart Sensor Protocol - SSP to exchange data and decide about the necessary requirements to fulfill the mission. As a result, the specified mission can be classified as: feasible, partially feasible or non-feasible. This phase is performed always when a different MOSA is connected and a new mission is specified. Missions can be adaptive and some configurations can change during the execution of a mission. 
2.1.8 The MOSA Architecture: The main feature of the MOSA architecture is the division of the system in two distinct modules, the aircraft module (the safety-critical part of the UAS) and the MOSA module (the non-safety-critical part of the UAS). MOSA systems include a set of embedded sensors that provide raw data for specific applications. In addition to the hardware, a MOSA system also includes the software necessary to carry out a mission, communicate with all sensors, and send/receive data to the aircraft. On-board processing reduces raw data complexity into ready-to-use information. Figure 1 shows a simplified functional diagram of the MOSA architecture and the interconnection among the system's components. The diagram can change in complexity and number of components according to a particular application.

MOSA systems make heavy use of Model-Driven Development (MDD). MDD is a software development methodology where the main elements are models, from which code is produced. MDD makes possible fast complex system prototyping, by the automatic generation of high performance code. This code can be embedded into electronic components to be applied to real time environments.

To communicate with the aircraft, the MOSA uses a standard interface, called SSP/SSI (Smart Sensor Protocol/Smart Sensor Interface). SSP is the communication protocol, while SSI is the interface that allows the MOSA system to use various services provided by the aircraft, particularly the air transportation service and communication with the GCS. MOSA systems can be used in different UAVs that had been adapted to communicate over the SSI/SSP. The communication protocol uses a plug-and-play mechanism to check if the aircraft is able to perform a specific mission. This possibility is negotiated between the MOSA payload and the UAV during the handshake phase of the protocol. In some cases, a longer range or better aircraft stability may be required, among other limiting factors. According to these limitations, MOSA systems must be able to accomplish, completely or partially a planned mission.

The MOSA approach leads to modern aerial systems that can accomplish complex missions, presenting decision-making capabilities and optimizing the air-to-ground, real-time dataflow within the limits of the communication channels. Although in complex systems, such as medium and large UAVs, hardware costs do not present a limitation, the use of MOSA can provide great versatility and flexibility in the development process of sensor systems for new applications. Different sensors and processing units can be integrated into the best cost/benefit sensor arrangement for a specific usage scenario.

The MOSA system was designed to automatically perform missions that can be pre-programmed at GCS. Besides that, missions can be reconfigured in case of events that can compromise the results of the mission or that degrades flight safety, such as an unexpected atmospheric condition change. IFA is responsible to signal MOSA about the necessity of a dynamic route modifications due degraded safety conditions. MOSA can dynamically choose the best sensor arrangement for a given atmospheric condition, mitigating the impact on mission results.

\section{CASE STUDY}

A reference implementation of a MOSA system for automatic mapping of sound sources activity on the ground is presented in this section. These sound sources include internal combustion engines and firearms activity, both related to illegal activities in preservation areas in Brazil.

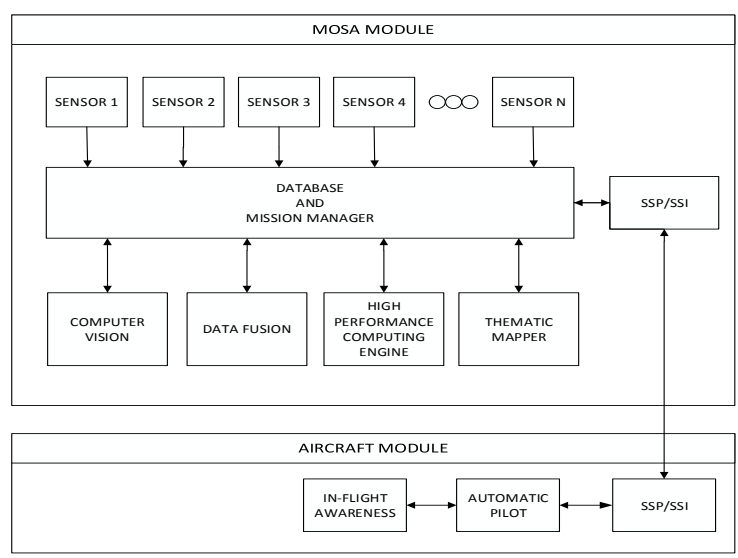

Figure 1. MOSA Functional Organization

\subsection{Environmental Monitoring Importance in Brazil}

Brazil has already destroyed $93 \%$ of the Atlantic Forest, most of the Araucaria Forest and part of Cerrado areas. The Amazon rainforest is the next environment under risk, suffering mostly from the substitution of the forest trees for grass, aimed at meat production, by soya beans production, hydroelectric energy generation, urban occupations, illegal wood poachers and miners.

On November 24, 2014, the newspaper Folha de São Paulo published a news entitled "Indians will use mobile phones in trees to monitor forests in Amazon" (Garcia, 2014), describing the importance of the use of the technology for environmental monitoring. This is just one of the many cases where continuous environmental monitoring is necessary.

There are biomes in Brazil with different compositions ranging from dense vegetation (rain forest) to sparse vegetation (savanna, cerrado, pampas). Aerial monitoring missions require different approaches to overcome difficulties, taking into account the particularities of each scenario. When a certain area needs to be monitored or supervised, two important issues must be addressed: poor geographical knowledge of the location and access difficulties to the region (Sá, 2002). Geographic knowledge of the area under investigation is essential for planning and accomplishment of the surveillance mission. The absence of updated cartographic documents makes it very difficult to locate targets.

In very wide areas of difficult access, it is often impractical to implement a continuous monitoring system, as there are cost and safety issues associated with the monitoring activities. A possible solution for these cases can be aerial-based monitoring. Aerial photography based on conventional aircraft is an expensive and time-consuming process when compared to the flexibility and versatility of recently available UAV platforms.

\subsection{Tracking Illegal Activities}

As a case study, to apply the concepts presented in this work, we describe in the next sections a MOSA system to monitor and track illegal activities in preservation areas focusing on the location and detection of human presence and medium-sized animals, gunshots and fires. The chosen area is the Brazilian cerrado. As can be seen in Figure 2, this is a type of biome similar to the African savannah, considering the techniques used for aerial monitoring. The very first implementation of a MOSA array in The Ranger Drone Project (Hemav Academics, 2014), was for surveillance of a savannah region. 

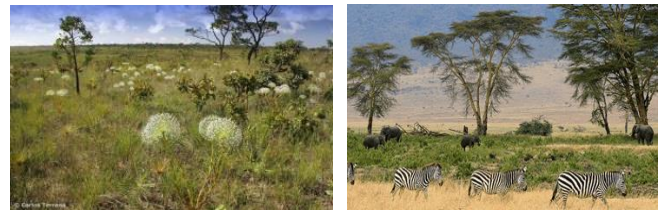

Figure 2. (left) Brazilian cerrado, (right) African savannah.

The described scenario usually is a poorly mapped area of difficult access, where there may be poaching, incidence of environmental crimes (such as illegal logging and silting of riverbeds), and even endangered species that need to be frequently monitored.

To plan a surveillance mission it is important to know:

(1) The types of data describing the phenomena / elements under study;

(2) The detection methods of the phenomena / elements that enable the selection of the sensors;

(3) The sensors selected.

In the context of this work, we address the following results:

- Map updates to reflect the cartographic reality of the area under monitoring;

- Automatic detection of gunshots, large animals and humans, characterized by sound and thermal emission;

- Animal movement and hunting activity: characterized by animal sounds, animal and human presence detected by thermal images and firearm activity.

\subsection{Organization of the Proposed System}

The system proposed in this paper consists of a Ground Sensor Network (GSN) integrated with the UAS and the MOSA system.

3.3.1 UAS and the MOSA System: The UAS chosen for this work is the Ararinha (Figueira, 2013), that can be seen in Figure 3 (left). It is an academic test platform for embedded systems used in many academics researches (gisa.icmc.usp.br). It is noteworthy its simplicity of construction, flight characteristics appropriate to this case study and the ease of operation. In addition, this project has autonomous flight capability and it is open source.
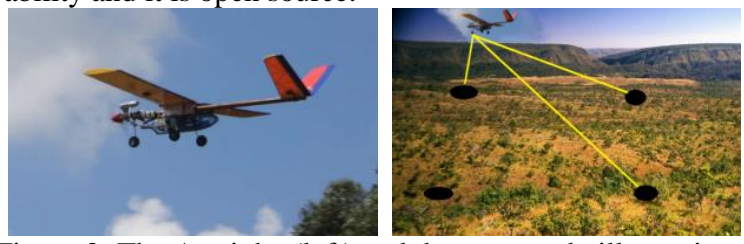

Figure 3: The Ararinha (left) and the case study illustration (right).

3.3.2 Ground Sensor Network: In the context of environment monitoring, the acquisition, processing and analysis of sounds are important since they may increase the perception of the phenomena that occur in a given area. Inspired by the Soundscape, which is the study of sound in a specific scenario (Pijanowski et al., 2011), embedded audio recorders could be used in multiple ground-based sensor stations to register occurring sounds in the monitored area. These stations can be connected wirelessly to form a Ground Sensor Network (GSN).
The GSN collect environment sounds, pre-process and send them (via a radio modem) to an UAV overflying the area. In the GSN, sound data are processed by Freire's method (Freire, 2014a) and sent to the UAV. This process reduces the volume of data over the limited bandwidth channel between the GSN and the UAV. The sound information, images and GPS coordinates are processed on-board, in the MOSA system.

The following elements, illustrated at Figure 3 (right), compose the system:

1) A GSN composed by microphone arrays and sound processors deployed in the geographical area of interest. Continuous processing of raw sound data results in time stamps, DOA vectors and sound classification;

2) UAV flights over the GSN area collecting the processed data;

3) On-board sound data processing, by the MOSA payload, to detect and locate targets. It is also possible to use algorithms to determine the angle of incidence of the sound and the source motion;

4) On-board processing of aerial thermal imaging for the detection of the presence of large animals (including humans) in the area;

5) On-board merging of the thematic information from the sound sensors with the thematic information obtained from the thermal sensor to extract the following information: presence of animals and humans; detection of poaching activity; detection of routine animal activity.

It must be understood that communication between the GSN and the MOSA payload is not always possible, since the UAV will not always be flying over the GSN. For example, ground sensors can record and store chainsaw sound signatures over a whole week, and these data will be sent to MOSA for analysis only when the UAV flies over the GSN.

\section{AUDIO SIGNAL PROCESSING}

Audio signal processing is done in ground stations equipped with microphone arrays (Brandstein and Ward, 2001). In a later stage, packets containing compressed, locally derived, information can be sent to an overflying UAV, which translates relative measurements into a global space and time system. Global events can be constantly reviewed and improved by new information, gathered from the ground stations.

While these methods have been implemented and tested, they have not yet, at the time of writing, been integrated in the UAV/MOSA platform, nor deployed for the proposed application on environmental monitoring. Furthermore, all local and global clocks are assumed to be synchronized.

\subsection{Communication between local stations and UAV}

Different types of (encrypted) data packets are exchanged between UAV and local stations. These are information packets and control packets. Information packets are sent from a ground station to a UAV/MOSA, and control packets are sent from the UAV/MOSA to a ground station.

4.1.1 Information packets: These are of two types: information about locally-recorded events and information about movement of local sensors.

A locally-recorded event is compressed in a packet containing: a direction-of-arrival relative to the array coordinate system, (i.e., a local direction); a timestamp of the moment the sound reaches the array (i.e., a local time); and a dictionary data structure providing classification of sound sources (for 
example: gunshot, speech, parrot, red-maned wolf, etc) to a tuple containing an estimate of the probability that the detected sound of interest was generated by that type of sound source and a measure of reliability of that estimate.

Information about movement is given by three Euler angles specifying rotation, one three-dimensional vector specifying translation, a timestamp for the beginning of the movement and a second timestamp for the end of movement.

4.1.2 Control packets: These are created by the MOSA system according to the mission, and are sent from the UAV/MOSA to a ground station. There are two types of control packets: request for information, and request for movement.

In the case of microphone arrays as ground stations, request for information may specify time of occurrence, direction of occurrence, type of occurrence, or any other combination. In the case of direction of occurrence specifications, a beam former (Brandstein and Ward, 2001) may execute locally, which "focuses" the sensing array on a given direction.

\subsection{Local Audio Signal Processing}

The tasks related to passive audio signal processing performed by local stations consists of sound source detection, localization and classification. The ground stations continuously process the incoming sound, and transmit compressed information to the UAV.

Microphone arrays (Brandstein and Ward, 2001) are used for synchronous signal acquisition. This choice of technology enables estimation of the DOA of the arriving acoustic energy, and beamforming is used to increase the SNR (Signal to Noise Ratio). In the case of transient sound signals, like gunshots, microphone array technology allows use of space-time methods for signal detection (Freire, 2014a, Freire, n.d.).

Sound source localization is performed by Generalized CrossCorrelation (GCC) (Knapp and Carter, 1976) methods. Timedelay Estimates (TDE's) are derived, for each microphone pair, as the time-argument that maximizes a GCC with Phase Transform (PHAT) weights (Knapp and Carter, 1976). A straightforward Least Squares (LS) implementation is given in (Caffery, 2000). However, significative improvements can be obtained over that implementation by employing an Iterative Least-Squares (ILS) algorithm (Bjorck, 1996), which effectively implements the concept of search for Spatially Coherent Subset of TDE's (SCST). This improved method and reasons why it performs well in low signal-to-noise ratio scenarios is discussed in (Freire, 2014b). Among the three methods: RAW LS (Caffery, 2000), WLS (Varma et al., 2002), and ILS (Freire, 2014b), ILS is the method of choice in this application.

Sound source classification is performed by Hidden Markov Models, (HMM, Rabiner and Juang, 1986) operating on Melfrequency cepstral coefficients (Mermelstein, 1976). An HMM is created for each sound class of interest, for example: gunshot, speech, automobile, bird from parrot, bem-te-vi, sabia, etc, redmaned wolf, etc. For selected windows, the HMM model of each class outputs a probability that the sound window was produced by that model.

Sound detection is generally implemented by establishing energy thresholds at various frequency bands, followed by HMM classification, and finally detecting if any of the HMM models outputs a probability above a threshold. However, for gunshots, in particular, the method described and tested in simulations in (Freire, 2014a) and tested on real firearms data in (Freire, n.d.) is preferred.

The ground stations may be capable of active audio processing, for example, of moving microphones within the array, or the translation the entire array. Moving single microphones of the array is a local process that does not need to be communicated to the UAV. Moving the entire array, however, needs to be communicated to the UAV, along with timestamps of movements, so that the UAV can track the array's position in the map, which is necessary for correctly performing local-toglobal event resolution. The movements of the entire array are coded as Euler angles (for specifying rotation) and threedimensional vectors (for specifying translations) (Paul, 1981) and need to be timestamped.

\subsection{Relativity in Acoustical Space and Time}

Because time and direction are relative measurements, a process of local-to-global event resolution needs to be carried out in the $\mathrm{UAV}$, once the information packets are received. This process takes into account the value of the speed of sound and the position and orientation of the microphone array at the moment of each of the audio captures.

4.3.1 Relativity of local time: Relativity of time in Acoustics is derived from the finitude of the speed of sound. If a sound of interest is detected in an array placed at position $p_{a}$ at time $t a$, then the time of the event $t$, at the source position $p_{s}$ is

$$
t_{s}=t_{a}-\frac{d\left(p_{a}, p_{s}\right)}{331.4+0.6 T_{C}}
$$

Where the denominator gives speed of sound as a function of temperature Tc in Celsius degrees, and $d$ gives the Euclidean distance between two points.

4.3.2 Relativity of local DOA: Direction-of-arrival, too, is relative to observer. The local stations transmit local information to the UAV/MOSA, which then translates them to a global coordinate system. A DOA is a vector starting at the array center and pointing in a given direction. If this vector is represented $\mathrm{pA}$ in the array coordinate system, it will be represented as $\mathrm{pHM}$ in the map (global) coordinate system. The mapping is performed by a homogeneous transformation,

$$
H: p_{A} \rightarrow p_{M}^{H}
$$

where

$$
p_{M}^{H}=R_{z}\left(\theta_{E}\right) R_{y}\left(\phi_{E}\right) R_{x}\left(\varphi_{E}\right) T\left(\Delta_{x}, \Delta_{y}, \Delta_{z}\right) p_{A}
$$

where

$\mathrm{Rw}, \mathrm{w}$ in $\{\mathrm{x}, \mathrm{y}, \mathrm{z}\}$ is the rotation matrix around the w-axis; $\theta \mathrm{E}$, $\varphi \mathrm{E}, \psi \mathrm{E}$ are the Euler angles; and $\mathrm{T}$ is the translation matrix.

\subsection{Global Event Binding}

Binding is the process of recognizing two seemingly distinct events as one. The UAV/MOSA collects various local information packets, converts them to a global space and time system, and binds them. The binding may span more than one sensory modality. In the case study presented in Section 4, for example, a gunshot signal is recorded by two microphone arrays and a camera, and binds all three relative perceptions into one. This section briefly discusses the geometry and the role of uncertainty in global event binding, considering sound and image sensors. 
Figure 4 shows single-sensory integration of two DOAs, captured by two arrays at different distances from the sources. Initially, DOA is considered as a line. Source is placed at the crossing of the two DOAs. Then, considering that increasing distance between microphone array and source deacreases SNR, by $6 \mathrm{~dB}$ per doubling of distance, the estimation error (in degrees) becomes a function of estimated distance between source and microphone array. Considering a cone for representing a DOA with uncertainty in estimation, the inner angle of this cone becomes larger with increasing distance (quantitative measurements related to this are reproduced in this paper, in Figure 6). The source can be placed somewhere in the intersection of the two DOA cones.

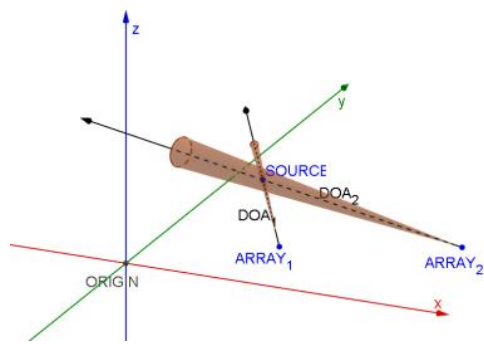

Figure 4. Estimated DOA's.

Global time of event occurrence at the source position may also be estimated from local times evaluated by the microphone arrays, and their respective distances to the source. Once this global time is known, along with global position, other local sensors may be queried for information: images from cameras facing that point, at that time (for light sources we approximate local time $=$ global time $)$, or from microphone arrays at corresponding tuples (position, time), where calculation of time takes into account the speed of sound.

\section{PRELIMINARY RESULTS}

\subsection{Implementation of the System}

The diagram in Figure 5 presents a Data Flow Diagram (DFD) of the MOSA payload. This DFD was implemented and functionally simulated as a Matlab Simulink Model.

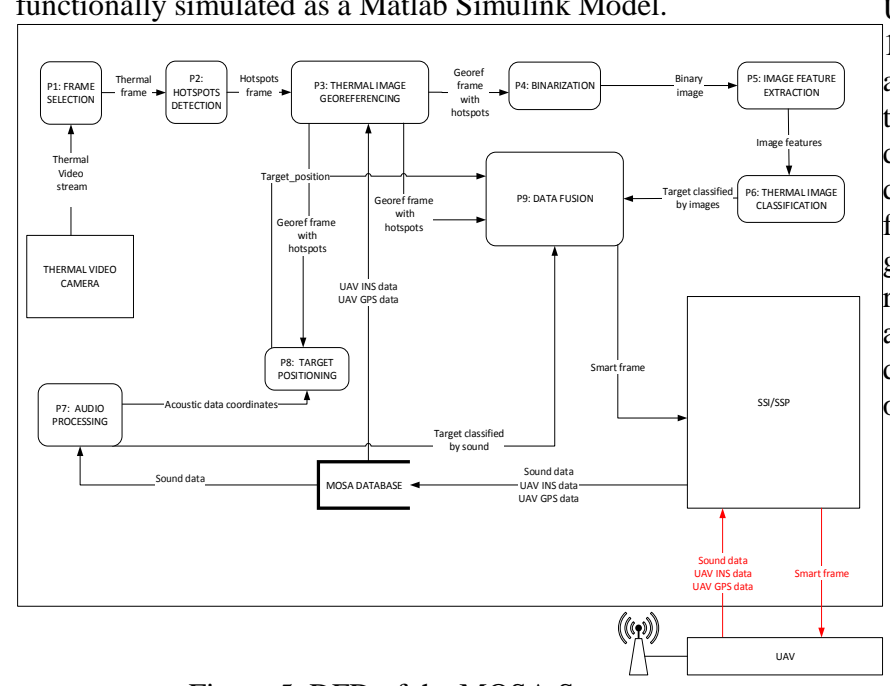

Figure 5. DFD of the MOSA System.

The processes in this DFD are:

P1: FRAME SELECTION: a process that receives a video stream $\mathrm{N}$ frames per second and separates periodic frames from the sequence, since there is a huge image overlap among adjacent frames in the time sequence;

P2: HOT SPOTS DETECTION: this process uses a search window to find clusters of pixels in thermal images that represent elements that have temperatures above a given threshold;

P3: THERMAL IMAGE GEOREFERENCING: process that correlates elements in the thermal images to coordinates from different sources (GPS, IMU and documents in the geographic database);

P4: BINARIZATION: process that converts an image into another image with two groups of pixels: cluster of hot spots and the rest of the image;

P5: IMAGE FEATURES EXTRACTION: process that analyzes binary image produced by $\mathrm{P} 4$ and extracts the contour of the cluster of pixels with high temperature;

P6: THERMAL IMAGE CLASSIFICATION: process that compares the temperature of the element contained in the binary image with a calibration table that contain the temperature function;

P7: AUDIO PROCESSING: continuous processing of DOA received from the GSN. The DOA, timestamps, and possible source classifications, linked to their respective probabilities, are sent to the global event database in the UAV.

P8: TARGET POSITIONING: a fusion process for the coordinates of targets calculated from the images and from sound processing;

P9: DATA FUSION (GLOBAL EVENT BINDING): When local information from various sources could plausibly refer to the same source event, data fusion, or event binding, occurs.

\subsection{Direction-of-arrival of gunshot signals}

Direction-of-arrival of gunshot signals is estimated by the method of (Freire, 2014b). Figure 6 is extracted from that paper and shows cumulative distribution functions of error (in degrees) of three different DOA estimation methods of the LSTDOA (Least Squares, Time Difference of Arrival) class: RAW of (Caffery, 2000), WLS of (Varma, 2002) and the ILS (Bjorck, 1996).

Under higher SNR, the DOA error is within 1.5 degrees for $100 \%$ of 1024 instances. For an SNR of $0 \mathrm{~dB}$ (gunshot signal and overall audio window excluding the gunshot itself having the same amount of energy), performance of RAW and WLS degrades significantly and ILS estimates correct DOA within 5 degrees of error, for approximately $90 \%$ of instances. This figure was obtained from simulation data, but data for real gunshot signals is available in (Freire, 2014c). The results with real data are not reviewed here, due to complexity of data analysis caused by lack of knowledge of the array's orientation during recording, however, they did corroborate the superiority of the ILS method for low SNR.

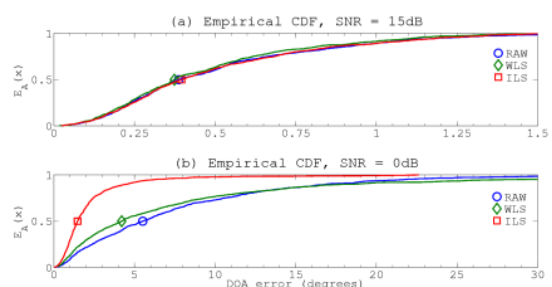

Figure 6: (Color online) Empirical CDF of error at (a) $\mathrm{SNR}=15$ $\mathrm{dB}$ and (b) $\mathrm{SNR}=0 \mathrm{~dB} . \mathrm{E}_{\mathrm{A}}(\mathrm{x})$ is the empirical $\mathrm{CDF}$ of error of algorithm A. For picture clarity, the curves may not include the highest 2\% sampled errors. Reproduced with permission from

(Freire, 2014b). Copyright 2014, Acoustical Society of America. 


\subsection{Detection of gunshot signals}

Detection is performed by the method first described in (Freire, 2014a), where it was tested using simulation data. A field report on detection of riffle shots is currently under review (Freire, n.d.), and has achieved perfect performance (100\% hits, 0 false positives) for detection of AGLC rifle gunshots in an open field environment like that of cerrado and savannah, at $670 \mathrm{~m}$ of distance between microphone array and gunshot, with prospective $95.36 \%$ recall and $3.74 \%$ false positive rate for double that distance.

\subsection{On-board Image Processing and Data Fusion}

Images are acquired by the thermal camera in the MOSA system mounted on the UAV. The images are inspected using specific algorithms that search for hot spots and classify targets by temperature thresholds. Based on the positioning data and platform attitude (coming from SSP / SSI) it is possible to georeference the thermal images with marked hot spots. As part of the cooperation agreement between the University of São Paulo and the Hemav Academic Team, composed by students of the Polytechnic University of Catalonia, some images of large mammals were processed using the proposed system. Some qualitative results are shown in Figure 7. Situational information is sent to the GCS containing the likely coordinates of targets and probable classifications for them. This information can be converted into themes of thematic maps using a reference GIS.

\section{DISCUSSION AND FUTURE WORKS}

The aim of this paper is to present the MOSA concept, under an architectural point of view, as a reference system to automatically produce thematic maps. The system embeds all hardware and software necessary to process raw data collected by an array of sensors. The key idea is to produce real time, ready to use thematic information transmitted over low bandwidth communication channels to a GCS. In the GCS, a Geographic Information System can be used to produce derived thematic maps.

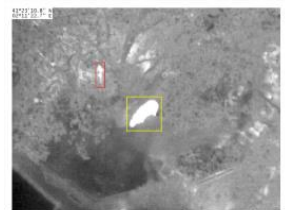

(a) A hyppo.

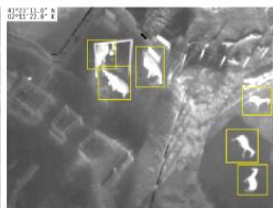

(b) Many animals

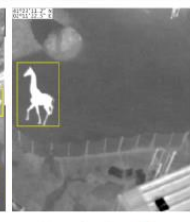

(c) A giraffe.
Figure 7: Thermal images processed by R_MOSA

The proposed MOSA architecture is described briefly to show the integration of all concepts involved in this paper. It comprises a plug-and-play mechanism: the Smart Sensor Interface and the Smart Sensor Protocol, allowing easy payload and aircraft interchange. Aircraft and payload communicate during the start-up of the system, exchanging mission requirements and flight parameters in order to define the feasibility, complete or partial, of the mission.

An application on Environmental Monitoring was discussed to make clear the more general MOSA architecture. A data flow diagram, including all processes to generate automatically a thematic map was proposed, showing its implementation feasibility.
Future works include some flight tests in a Brazilian military scenario to validate performance aspects. These tests will eventually lead to some changes in the DFD presented in Figure 5. First results encourage further development showing that MOSA can represent a big step towards better usability of UAVs in most applications.

\section{CONCLUSIONS}

Several MOSAS are under development at the time of this writing. Most of them are simple systems with proven results from previous developments. The main task in these cases is the automation of some processes and changes in some others in order to comply with the MOSA architecture. On the other hand, the automatic generation of thematic maps is a quite complex system where we can work out most aspects of the MOSA architecture.

This work was structured based on association between the MOSA and a collaborative GSN. The feasibility of using microphones arrangements embedded in UAVs for the detection and localization of sounds was proven in Basiri et al. (2012).

In addition to the environmental monitoring it is possible to implement the proposal of this work the following scenarios:

- Soundscape ecology;

- Search and rescue people;

- Disaster Monitoring;

- Urban Surveillance.

\section{ACKNOWLEDGEMENTS}

The authors acknowledge the support granted by CAPES, Institute of Computing and Computational Mathematics from the University of São Paulo (ICMC-USP), Department of Computer Science from the Minas Gerais Federal University, Brazilian Army, Geomatic Division from the Centre Tecnològic de Telecomunicacions de Catalunya (GD - CTTC), Spain and Hemav Academic Team.

\section{REFERENCES}

Adamchuk, V. I., Hummel, J., Morgan, M. and Upadhyaya, S., 2004. On-the-go soil sensors for precision agriculture. Computers and electronics in agriculture 44(1), pp. 71-91.

Albino, V., Berardi, U. and Dangelico, R. M., 2015. Smart cities: Definitions, dimensions, performance, and initiatives. Journal of Urban Technology.

Austin, R., 2010. Unmanned aircraft systems: UAVS design, development and deployment. Wiley \& Sons, 2010, 332p.

Basiri, M., Schill, F., Lima, P. U., Floreano, D., 2012. Robust acoustic source localization of emergency signals from Micro Air Vehicles. IROS 2012: 4737-4742.

Bjorck, A., 1996. Numerical methods for least squares problems.Society for Industrial and Applied Mathematics (SIAM), North-Holland, Amsterdam.

Brandstein, M. and Ward, D., 2001. Microphone arrays: signal processing techniques and applications. Springer Verlag.

Caffery, J., 2000. A new approach to the geometry of TOA location. In: Proceedings of the 52nd Vehicular Technology 
Conference (IEEE-VTS Fall VTC 2000), Vol. 4, IEEE, Boston, MA, EUA, pp. 1943-1949.

Figueira, N. M., 2013. Mission-oriented sensor arrays - An approach towards UAS usability improvement in practical applications. In: Proceedings of the 5th European Conference for Aeronautics and Space Science (EUCASS), Munich, Germany, pp. 149-156.

Freire, I. L., 2014a. Detection of impulsive signals by timeseries of direction-of-arrival features. In: Proceedings of the $X X$ Congresso Brasileiro de Automatica, SBA, Belo Horizonte, Minas Gerais, Brazil, pp. 149-156.

Freire, I. L., 2014b. Robust direction-of-arrival by matchedlags, applied to gunshots. The Journal of the Acoustical Society of America 135(6), pp. EL246-EL251. http://dx.doi.org/10.1121/1.4874223.

Freire, I. L., 2014c. Localização e detecção de caçadores no espaço-tempo acústico coerente. (Localization and detection of sniper in the coherent acoustical space-time) $\mathrm{PhD}$ Thesis. Instituto Militar de Engenharia, Brazil.

Freire, I. L., n.d. Gunshot detection in acoustical space-time: analysis of experimental data. Submitted to Applied Acoustics (under review).

Garcia, R., 2014. Índios usarão celulares em árvores para monitorar florestas no Pará. Folha de São Paulo, São Paulo, 24 nov 2014. caderno Ambiente. http://www1.folha.uol.com.br/ambiente/2014/11/1552209indios-usarao-celulares-em-arvores-para-vigiar-floresta-nopara.shtml. Acessed 12 april 2015.

Grenzdorffer, G., Engel, A. and Teichert, B., 2008. The photogrammetric potential of low-cost UAVs in forestry and agriculture. The International Archives of the Photogrammetry, Remote Sensing and Spatial Information Sciences 31(B3), pp. 1207-1214.

Hemav Academics, 2014. Ranger Drone Project. http://hemav.com/academics/. Acessed 17 may 2015.

Knapp, C. and Carter, G. C., 1976. The generalized correlation method for estimation of time delay. Acoustics, Speech and Signal Processing, IEEE Transactions on 24(4), pp. 320-327.

Longuitano, G. A, 2010. VANTS para sensoriamento remoto: aplicabilidade na avaliação e monitoramento de impactos ambientais causados por acidentes com cargas perigosas. Tese de doutorado, USP.

Machado, P. A.L., 1995. Direito ambiental brasileiro. $5^{\text {a }}$ ed. São Paulo: Malheiros Editores, 696p.

Marsal-Llacuna, M. L., Colomer-Llinas, J. and MelendezFrigola, J., 2015. Lessons in urban monitoring taken from sustainable and livable cities to better address the smart cities initiative. Technological Forecasting and Social Change 90, pp. 611-622.

Mattei, A. P., Fonseca, E., Figueira, N. M., Trindade, O., e Vaz, F., 2013. UAV In-Flight Awareness: A Tool to Improve Safety. In: 5TH European Conference for Aeronautics and Space Sciences (EUCASS), Munich.
Mermelstein, P., 1976. Distance measures for speech recognition, psychological and instrumental. Pattern Recognition and Artificial Intelligence 116, pp. 374-388.

Paul, R., 1981. Robot manipulators: mathematics, programming, and control: the computer control of robot manipulators. The MIT Press.

Pijanowski, B. et al., 2011. Soundscape Ecology: The Science of Sound in the Landscape. BioScience, Vol. 61, No. 3 (March 2011), pp. 203-216. doi: 10.1525/bio.2011.61.3.6.

Pires, R. M., Chaves, A. A. and Branco, K. R., 2014. Smart sensor protocol-a new standard for UAV and payload integration. In: Unmanned Aircraft Systems (ICUAS), 2014 International Conference on, IEEE, pp. 1300-1310.

Rabiner, L. and Juang, B.-H., 1986. An introduction to Hidden Markov Models. IEEE ASSP Magazine 3(1), pp. 4-16.

Rodrigues, D., J. C. Estrella, M. Vieira, J. B. Camargo Jr, K. R. L. J. C. Branco, and O. Trindade Jr., 2011. Service-oriented architectures for complex safety-critical embedded systems: a case study on UAVs," in Proc. I Brazilian Conference on Critical Embedded Systems - CBSEC, S〜 ao Carlos-SP, Brazil, p. 130.

Rosnell, T., Honkavaara, E. and Nurminen, K., 2011. On geometric processing of multi-temporal image data collected by light UAV systems. Int. Arch. Photogramm. Remote Sens. Spat. Inf. Sci 38, pp. 1-6.

Sá I. B., 2002. Monitoramento ambiental: a degradação ambiental no trópico semi-árido do Nordeste brasileiro. In: Simpósio Regional de Geoprocessamento e Sensoriamento Remoto, Aracaju. Anais. Aracaju: Embrapa Tabuleiros Costeiros. Acessed 12 April 2015.

Schmale, I., David, G., Dingus, B. R. and Reinholtz, C., 2008. Development and application of an autonomous unmanned aerial vehicle for precise aerobiological sampling above agricultural fields. Journal of Field Robotics 25(3), pp. 133 147.

Tokekar, P., Vander Hook, J., Mulla, D. and Isler, V., 2013. Sensor planning for a symbiotic UAV and UGV system for precision agriculture. In: Intelligent Robots and Systems (IROS), 2013 IEEE/RSJ International Conference on, IEEE, pp. $5321-5326$

Trindade Jr, O. et al, 2010. A Layered Approach to Design Autopilots. In: International Conference on Industrial Technology (ICIT), 2010, Santiago, Chile. IEEE Press, 2010, v. v1, p. 1395-1400.

Varma, K., Ikuma, T. and Beex, A., 2002. Robust TDE-based DOA estimation for compact audio arrays. In: Sensor Array and

Multichannel Signal Processing Workshop Proceedings (SAM 2002), Rosslyn, VA, USA, pp. 214-218. 